\title{
A source of terahertz coherent phonons
}

\author{
Sergio S Makler $\dagger^{+}$, M I Vasilevskiył*, E V Anda§, D E Tuyarot $\|$, \\ J Weberszpil † and H M Pastawski $\uparrow$ \\ $\dagger$ Instituto de Fisica, Universidade Federal Fluminense, Campus da Praia Vermelha, \\ Avenida General Milton Tavares de Souza s/n, 24210-340-Niterói, RJ, Brazil \\ $\ddagger$ Faculty of Applied Physics, Nizhni Novgorod University, Nizhni Novgorod, 603600, Russia \\ $\S$ Departamento de Fisica, Pontificia Universidade Católica do Rio de Janeiro, Caixa Postal \\ 38071, 22452-970, Rio de Janeiro, RJ, Brazil \\ || Instituto de Fisica, Universidade Federal do Rio de Janeiro, Caixa Postal 68.528, 21945-970, \\ Rio de Janeiro, RJ, Brazil \\ ๆ Facultad de Matemática, Astronomia y Fisica, Universidad Nacional de Córdoba, Ciudad \\ Universitária, 5000 Córdoba, Argentina
}

Received 13 October 1997, in final form 17 March 1998

\begin{abstract}
Resonant tunnelling in a double-barrier heterostructure (DBHS) designed in order to produce an intense coherent emission of phonons is studied theoretically. This emission is achieved when the energy difference between the first excited level and the ground state in the well is close to the LO-phonon energy. The LO phonons emitted during the electron transition decay into a pair of ( $\widetilde{\mathrm{LO}}$ and TA) phonons that leave the well in the [111] direction. These secondary TA phonons are coherent and form a beam that we call a SASER (by analogy with 'laser'). The circulating current and the populations of phonons and electrons in the well are calculated by solving appropriate kinetic equations. Some possible applications of this system are discussed.
\end{abstract}

\section{Introduction}

In recent years, the number of studies of mesoscopic systems has steadily grown. New theoretical approaches appeared at the time when new technologies were developed to produce systems of sizes ranging from $5 \mathrm{~nm}$ to $500 \mathrm{~nm}$. In particular, doublebarrier heterostructures (DBHS) have attracted a lot of attention due to their potential applications as ultra-high-frequency electronic oscillators, diodes, transistors and other electronic devices [1].

Following the work of Goldman, Tsui, Cunningham and others [2-4], the importance of electron-phonon interaction as regards the electronic properties of these structures was realized. Due to the polar character of GaAs and other III-V compounds, the most important scattering process for electrons inside the well is that due to LO phonons. Several studies have taken into account the effect on the electronic current of electron-phonon interaction in the system [5-9]. However, little priority has been given to the study of the phonons generated in this process, the way they propagate, their decay processes etc.

+ E-mail: sergio@if.uff.br.

* Present address: Departamento de Fisica, Escola de Ciências, Universidade do Minho, Largo do Paço, 4709 Braga Codex, Portugal. 
This paper is dedicated mainly to the study of phonon generation in a DBHS experiencing the effect of an external applied bias. The LO phonons have a very flat dispersion relation for the wavevector with which they are generated. Therefore they have a very small group velocity. Also, if the LO-phonon frequency in the well lies in the gap for phonons in the barriers, they are confined inside the well $[10,11]$. Finally, their lifetime is short since they decay due to anharmonicity into a pair of phonons, $\mathrm{LO} \rightarrow \widetilde{\mathrm{LO}}+\mathrm{TA}$ [12]. The TA phonons have a very long lifetime [13] and a mean free path of $\sim 2 \mathrm{~mm}$ [14]. These secondary TA phonons are our main interest in this work because they form an intense coherent beam that is potentially useful in several applications.

Generation of coherent phonons was discussed theoretically in the early 1960s [15], and was observed experimentally at almost the same time [16, 17]; also a variety of possible experiments and applications were suggested [18]. These phonons were generated by intense laser pulses. On the other hand, generation of phonons by stimulated emission was also considered theoretically [19]. The amplification of sound by stimulated emission has already been observed experimentally. Prieur et al [20,21] studied the phonon emission due to transitions in a two-level system well known in glasses. These phonons had a wide energy distribution around $\epsilon=0.01 \mathrm{meV}$. In [20] the authors used the term SASER for a device that could produce a coherent phonon beam. In another paper referring to coherent sound of lower energy [22], acoustic phonons were optically generated in a superlattice, in such a way that their wavelengths were the same as the period of the superlattice (i.e., $500 \AA$ ). Recently [23] the generation of high-frequency monochromatic acoustic waves by laserinduced thermomodulation was reported. The frequency of the phonons ranged from 2 to $4 \mathrm{GHz}$, in comparison with $2 \mathrm{THz}$ for our device. More recently, Zavtrak and coworkers [24-29] proposed a device (called a saser in [29]) formed by a dielectric liquid with small particles or gas bubbles, working similarly to a free-electron laser. That device could produce coherent acoustic phonons by stimulated emission at a frequency $\omega \approx 2 \mathrm{kHz}$. Fokker et al [30,31] report experimental results on stimulated emission of phonons in an acoustic cavity, obtained using the metastable Zeeman-split doublet in ruby. They claim that this is a first step toward constructing a phonon laser. Their phonons have frequencies of about $60 \mathrm{MHz}$. Pulses of coherent THz optical phonons have been produced by femtosecond-pulsed lasers. For more details see the review by Merlin [32] and references therein.

The main difference between the devices in the studies cited above and the one proposed here is that the DBHS resonator could produce a continuous beam of coherent phonons in the region of $2 \mathrm{THz}$. The ultra-short wavelength of these phonons permits potential applications that will be discussed at the end of this article.

The paper is organized as follows. In section 2 we present the main features of the device and the basic principle of its operation. In section 3 we establish the kinetic equations that govern the evolution of the electron and phonon populations inside the well, and we describe the calculation of the relevant parameters involved in the problem. The following section, section 4 , is devoted to discussion of the calculated results, of some potential applications of the device, of the ways in which calculations related to it could be improved, and also to conclusions.

\section{The device}

The effect studied here could be present in a wide variety of DBHS systems. We discuss here the particular case of a structure made of GaAs-AlGaAs because it is simple to grow it by MBE. The system has to be tailored to produce an intense phonon emission. The 


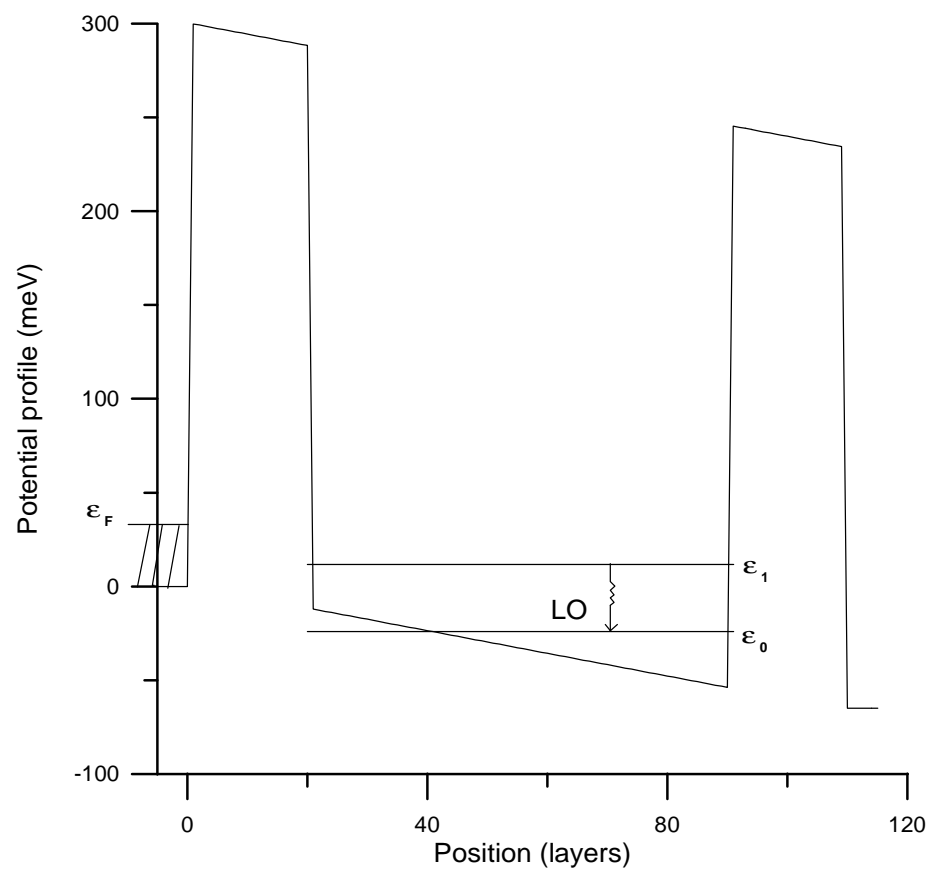

Figure 1. The potential profile and energy levels for the resonant condition.

energy difference $\Delta \epsilon=\epsilon_{1}-\epsilon_{0}$ increases slowly when the applied bias $V$ is augmented. The system is designed such that for a given $V, \epsilon_{1}>\epsilon_{F}^{L}$ and $\Delta \epsilon \lesssim \hbar \omega_{0}$. Here $\epsilon_{0}$ is the energy of the ground state in the well, $\epsilon_{1}$ is the energy of the first excited level, and $\omega_{0}$ is the $\Gamma$-point LO-phonon frequency. If the Fermi level $\epsilon_{F}^{L}$ at the emitter is low enough, then the ground state falls below the bottom of the conduction band and the current is almost suppressed until the level $\epsilon_{1}$ reaches $\epsilon_{F}^{L}$. On further increasing $V$, the current begins to flow through the excited level, but since $\Delta \epsilon$ remains much less than $\hbar \omega_{0}$, phonon emission is inhibited. For a given bias $V$, the resonant condition $\Delta \epsilon \approx \hbar \omega_{0}$ is achieved and the electrons begin to decay to the ground state by emitting LO phonons. The potential profile and the level positions for this resonant condition are shown in figure 1. As our system has wide barriers, the electrons remain confined in the well for long time $\left(\tau_{0}\right)$. When the $\mathrm{Al}$ concentration is greater than 0.25 [10] or 0.3 [11] the LO phonons are also confined inside the well. They can be also absorbed by electrons being excited from $\epsilon_{0}$ to $\epsilon_{1}$. This process acts in parallel with the decay of primary phonons due to anharmonicity.

We have calculated the transition rates of the emission and absorption processes following the evaluation of the electron-phonon interaction given by Trallero-Giner and Comas [33], and we show that the situation in which $\Delta \epsilon>\hbar \omega_{0}$ that occurs for higher applied bias is still compatible with energy and momentum conservation in the process of LO-phonon emission. The tunnelling current and the phonon emission continue to occur until the excited level is below the bottom of the conduction band in the emitter.

We have studied a DBHS at zero temperature experiencing the effect of an external potential $V$. When the barriers have the sizes used in our calculation, i.e., $b_{L}=b_{R}=56.5 \AA$, corresponding to 20 layers of $\mathrm{Ga}_{1-x} \mathrm{Al}_{x} \mathrm{As}$, with a height of $300 \mathrm{meV}$, the widths of the levels involved in the process, as is shown below, are about $\Gamma_{0}=0.005 \mathrm{meV}$ and $\Gamma_{1}=0.030 \mathrm{meV}$, depending slightly on $V$. It can be seen that the level positions $\epsilon_{0}$ and $\epsilon_{1}$ depend upon the 
well width $d$, the barrier widths $b_{L, R}$ and the barrier height $V_{0}$, and the applied bias $V$. For $d=197.8 \AA$, corresponding to 70 layers of GaAs grown in the [100] direction, we found that when the applied bias varies between $59.5 \mathrm{mV}$ to $90.7 \mathrm{mV}$, the position of the first excited level goes down from about $15 \mathrm{meV}$ to zero. Simultaneously the energy difference $\Delta \epsilon$ increases from $35.2 \mathrm{meV}$ to $37.9 \mathrm{meV}$. That means that for a device prepared with a Fermi level $\epsilon_{F}^{L}=15 \mathrm{meV}$, the system would have electrons injected into the excited level when $V$ is within the range given above. This includes the resonant value $\Delta \epsilon=36.6 \mathrm{meV}$.

\section{The calculation}

We are interested to know whether the coherence condition could be fulfilled, i.e., whether the number of secondary phonons inside the well could be much greater than one and so cause the stimulated decay of primary LO phonons [12]. When the field of acoustic phonons stimulates the decay, new phonons are generated in phase with the exciting field. As a consequence, the acoustic phonon beam will be coherent.

\subsection{The kinetic equations}

In this section, we establish a system of kinetic equations that govern the balance of the electron and phonon populations inside the well, which can be considered as a reaction chamber.

Inside the well, long-wavelength optical phonons can be classified as LO and TO confined modes and interface phonons. These latter have four branches, which, for a symmetric $\mathrm{AlGaAs} / \mathrm{GaAs} / \mathrm{AlGaAs}$ DBHS, are the GaAs-like symmetric $\left(\mathrm{S}^{-}\right)$and antisymmetric $\left(\mathrm{A}^{-}\right)$modes, and the corresponding AlGaAs-like modes $\mathrm{S}^{+}$and $\mathrm{A}^{+}$[36].

The relative strength of the interactions between these modes and electrons depends upon $\boldsymbol{q}_{\perp} d$, where $\boldsymbol{q}_{\perp}$ is the phonon wavevector perpendicular to the current and $d$ is the well width. It can be seen from the energy and momentum conservation relations that, close to the resonance, $\boldsymbol{q}_{\perp}$ is small. For small values of $\boldsymbol{q}_{\perp} d$, the interaction between electrons and interface phonons can be important for DBHSs with rather thin wells (as, for example, in the case of reference [3], where the satellite phonon peak in the characteristic curve was observed by the first time). Our device has a relatively thick well (about four times as thick as that of reference [3]). Since the relative importance of the interface phonons decreases strongly when the well width $d$ increases, the effect of these phonons on the electron dynamics should be negligible in our case. This is further confirmed by the results [34] of a calculation of the emission rates of confined LO and interface phonons. Moreover, since it is well known (see, e.g., [35]) that electron-TO-phonon interaction is much weaker than the Fröhlich interaction, we restrict ourselves to studying the interaction between electrons and just confined LO phonons.

To introduce the approximations and the notation used to derive these balance equations, we present first a rough estimate of the LO-phonon emission rate $w$ obtained by assuming simple electronic wavefunctions for the ground and excited states of an infinite rectangular well and a similar situation for the confined LO-phonon modes.

More accurate calculations for phonons in heterostructures can be found in the literature. Huang and Zhu [37] proposed macroscopic boundary conditions for displacements and potentials associated with phonon modes, which agree well with more reliable microscopic models [38-40]. However, we will show that the description of the generation of the acoustic phonon beam does not really require a precise calculation of the LO-phonon emission rate. The simplified model presented here is sufficient for our purposes and permits us to obtain 
relatively simple analytical expressions.

For the sake of simplicity, we impose the condition that the LO phonons are completely confined in the well by assuming the boundary conditions $u_{z}(z=0)=u_{z}(z=d)=0$, where we have taken the $z$-axis along the current direction with its origin at the end of the left-hand barrier and $d$ is the well width. To calculate the rate of transition between the electronic ground state $|0\rangle$ and the first excited state $|1\rangle$, according to the Fermi golden rule, we evaluate the matrix element $\left\langle 0\left|H_{e-p}\right| 1\right\rangle$. We can write

$$
\left\langle 0\left|H_{e-p}\right| 1\right\rangle=\left(\frac{\pi \hbar e^{2} \omega_{0}^{2}}{\epsilon^{*} \omega_{q}^{3} V}\right)^{1 / 2} \frac{1}{m^{*}} M_{01} b_{q}^{\dagger}
$$

where $b_{q}^{\dagger}$ is the creation operator for LO phonons with wavevector $\boldsymbol{q}$ and the effective dielectric constant is defined by $1 / \epsilon^{*}=1 / \epsilon_{\infty}-1 / \epsilon_{0}$. The transition matrix element is

$$
M_{01}=\frac{\hbar}{\mathrm{i}} \int \mathrm{d}^{3} r C_{q}^{+} \mathrm{e}^{-\mathrm{i} q_{\perp} \cdot r_{\perp}} \cdot\left[\Psi_{0}^{*} \nabla \Psi_{1}-\Psi_{1} \nabla \Psi_{0}^{*}\right]
$$

where

$$
\boldsymbol{C}_{q}=\frac{1}{q}\left[\boldsymbol{q}_{\perp} \cos \left(q_{z} z\right)+\mathrm{i} \boldsymbol{q}_{z} \sin \left(q_{z} z\right)\right]
$$

Here $\boldsymbol{q}_{z}$ and $\boldsymbol{q}_{\perp}$ are the components of $\boldsymbol{q}$ along and perpendicular to the current direction respectively and $q_{z}=r \pi / d$ where $r$ is an integer. The situation described here is in good agreement with experimental data [10].

By separating variables, writing $\Psi_{i}(\boldsymbol{r})=\psi_{i}(z) \mathrm{e}^{\mathrm{i} k_{\perp} \cdot r_{\perp}}$, and integrating over the transverse coordinates we get

$$
M_{01}=\hbar(2 \pi)^{2} \delta\left(\boldsymbol{k}_{1 \perp}-\boldsymbol{k}_{0 \perp}-\boldsymbol{q}_{\perp}\right)\left[\frac{q_{z}}{q} I_{1}-\frac{\boldsymbol{q}_{\perp} \cdot\left(\boldsymbol{k}_{1 \perp}+\boldsymbol{k}_{0 \perp}\right)}{q} I_{2}\right]
$$

where $\boldsymbol{k}_{1 \perp}$ and $\boldsymbol{k}_{0 \perp}$ correspond to the moment components of the initial and final electronic states, perpendicular to the current,

$$
I_{1}=\int \mathrm{d} z \sin \left(q_{z} z\right)\left[\psi_{0}^{*} \frac{\partial \psi_{1}}{\partial z}-\psi_{1} \frac{\partial \psi_{0}^{*}}{\partial z}\right]
$$

and

$$
I_{2}=\int \mathrm{d} z \cos \left(q_{z} z\right) \psi_{0}^{*} \psi_{1}
$$

From (1) and (4), we can express the rate of transition from the state $|1\rangle$ with wavevector $\boldsymbol{k}_{1 \perp}$ to the state $|0\rangle$ with wavevector $\boldsymbol{k}_{0 \perp}$ effected by emitting a phonon as

$$
\begin{aligned}
W\left(\boldsymbol{k}_{1 \perp}, \boldsymbol{k}_{0 \perp}\right)= & \sum_{\boldsymbol{q}} w_{+} \\
= & \frac{(2 \pi \hbar)^{2} e^{2} \omega_{0}^{2}}{2 \epsilon^{*} m^{*^{2}} V} \sum_{q_{z}} \int \mathrm{d} \boldsymbol{q}_{\perp} \frac{\left[q_{z} I_{1}-\boldsymbol{q}_{\perp} \cdot\left(\boldsymbol{k}_{1 \perp}+\boldsymbol{k}_{0 \perp}\right) I_{2}\right]^{2}}{q^{2} \omega_{\boldsymbol{q}}^{3}} \delta\left(\boldsymbol{k}_{1 \perp}-\boldsymbol{k}_{0 \perp}-\boldsymbol{q}_{\perp}\right) \\
& \times\left[n_{\mathrm{LO}}(\boldsymbol{q})+1\right] \delta\left(\Delta \epsilon-\hbar \omega_{0}+b\left(q_{z}^{2}+\boldsymbol{q}_{\perp}^{2}\right)+\frac{\hbar^{2}}{2 m^{*}}\left(k_{1 \perp}^{2}-k_{0 \perp}^{2}\right)\right)
\end{aligned}
$$

where we have used the well known quadratic dispersion law for LO phonons

$$
\omega_{q}^{2}=\omega_{0}^{2}-v^{2} q^{2} .
$$

As $v q / \omega_{0} \ll 1$ because $q \sim \pi / d$, we have used the approximation $\hbar \omega_{q} \simeq \hbar \omega_{0}-b q^{2}$ with $b=\hbar v^{2} / 2 \omega_{0}$. 
The integration over $\boldsymbol{q}_{\perp}$ is now easily performed and we get

$$
\begin{aligned}
W\left(\boldsymbol{k}_{1 \perp}, \boldsymbol{k}_{0 \perp}\right)= & \frac{(2 \pi)^{2} e^{2} \omega_{0}^{2}}{\epsilon^{*} m^{*^{2} V}} \sum_{q_{z}} \frac{\left[q_{z} I_{1}-\left(k_{1 \perp}^{2}-k_{0 \perp}^{2}\right) I_{2}\right]^{2}}{q_{0}^{2} \omega_{\boldsymbol{q}_{0}}^{3}}\left[n_{\mathrm{LO}}\left(\boldsymbol{q}_{0}\right)+1\right] \\
& \times \delta\left(\frac{2 m^{*}}{\hbar^{2}} \delta \epsilon+\left[(1+\eta) k_{1 \perp}^{2}-(1-\eta) k_{0 \perp}^{2}-2 \eta k_{0 \perp} k_{1 \perp} \cos (\varphi)\right]\right)
\end{aligned}
$$

where $\boldsymbol{q}_{0} \equiv\left(\boldsymbol{k}_{1 \perp}-\boldsymbol{k}_{0 \perp} ; q_{z}\right), \varphi$ is the angle between $\boldsymbol{k}_{0 \perp}$, and $\boldsymbol{k}_{1 \perp}, \delta \epsilon \equiv \Delta \epsilon-\hbar \omega_{0}+b q_{z}^{2}$, and $\eta=2 m^{*} b / \hbar^{2}$ is an adimensional parameter related to the curvature of the dispersion relation. We can estimate for GaAs that $\eta \sim 10^{-4}$. Therefore we retained only terms of zero order in $\eta$. In this case the zero of the argument of the Dirac function occurs when

$$
k_{0 \perp}=\tilde{k}_{1 \perp} \equiv \sqrt{k_{1 \perp}^{2}+\frac{2 m^{*} \delta \epsilon}{\hbar^{2}}} .
$$

If we assume that phonons are at thermal equilibrium and that their number (due to the small dispersion of the LO phonon branches) does not depend upon $\boldsymbol{q}$, the total scattering rate can be written as

$$
w\left[n_{\mathrm{LO}}+1\right]=\sum_{\boldsymbol{k}_{0 \perp}} W\left(\boldsymbol{k}_{1 \perp}, \boldsymbol{k}_{0 \perp}\right)
$$

where $n_{\mathrm{LO}}$ is the total number of LO phonons in the well. After performing the integration over $\boldsymbol{k}_{0 \perp}$, we obtain

$$
w=\frac{\pi e^{2} S}{\epsilon^{*} \omega_{0} m^{*} V} \sum_{q_{z}} \frac{\theta\left(\delta \epsilon+\hbar^{2} k_{1 \perp}^{2} /\left(2 m^{*}\right)\right)\left[q_{z} I_{1}+\left(2 m^{*} \delta \epsilon / \hbar^{2}\right) I_{2}\right]^{2}}{\sqrt{\left(q_{z}^{2}+\left(k_{1 \perp}-\tilde{k}_{1 \perp}\right)^{2}\right)\left(q_{z}^{2}+\left(k_{1 \perp}+\tilde{k}_{1 \perp}\right)^{2}\right)}}
$$

where $S$ is the device area.

By taking for $\psi_{0}$ and $\psi_{1}$ the results corresponding to the infinite well

$$
\psi_{0}=\sqrt{\frac{2}{d}} \sin \frac{\pi z}{d} \quad \text { and } \quad \psi_{1}=\sqrt{\frac{2}{d}} \sin \frac{2 \pi z}{d}
$$

we get

$$
I_{1}= \begin{cases}-\frac{3}{2} \frac{\pi}{d} & \text { for } r=1 \\ -\frac{1}{2} \frac{\pi}{d} & \text { for } r=3 \\ 0 & \text { otherwise }\end{cases}
$$

and

$$
I_{2}= \begin{cases}\frac{1}{2} & \text { for } r=1 \\ \frac{1}{4} & \text { for } r=3 \\ 0 & \text { otherwise. }\end{cases}
$$

We can see that $w$ is a function not only of the electronic energy difference but also of $k_{1 \perp}$. In the first approximation, it is sufficient to retain only terms with $r=1$.

We turn now to the three-dimensional kinetic equations. The rates of the electron and phonon transitions depend upon the momenta perpendicular to the current, $\boldsymbol{k}_{0 \perp}, \boldsymbol{k}_{1 \perp}$, and $\boldsymbol{q}_{\perp}$. We are assuming here that the wavefunctions of the electrons and LO phonons inside the well have well defined $z$-parts (corresponding to $k_{0 z}$ at the emitter, for the ground state 
etc). In fact the incomplete confinement of the modes in the $z$-direction produces a small spread of the $z$-component of the wavevector that is of the order of $\Delta k_{z 0} / k_{z 0} \sim 10^{-4}$. This spread is neglected in our calculation.

The rate of increase of the number of electrons injected into the excited state is

$\frac{\mathrm{d} n_{1}\left(\boldsymbol{k}_{1 \perp}\right)}{\mathrm{d} t}=G\left(\boldsymbol{k}_{1 \perp}\right)-\sum_{\boldsymbol{q}_{\perp} \boldsymbol{k}_{0 \perp}}\left[w_{+} n_{1}\left(\boldsymbol{k}_{1 \perp}\right)-w_{-} n_{0}\left(\boldsymbol{k}_{0 \perp}\right)\right]-R_{1}\left(\boldsymbol{k}_{1 \perp}\right) n_{1}\left(\boldsymbol{k}_{1 \perp}\right)$

where $G\left(k_{1 \perp}\right)$ is the rate at which electrons enter from the left-hand barrier with perpendicular momentum $\boldsymbol{k}_{1 \perp}$ (i.e., the current spectral density), and $n_{0}\left(\boldsymbol{k}_{0 \perp}\right)$ and $n_{1}\left(\boldsymbol{k}_{1 \perp}\right)$ are the electron population distributions. $R_{1}\left(k_{1 \perp}\right)$ is the rate of escape of electrons through the right-hand barrier, and $w_{+}$and $w_{-}$stand for the emission and absorption rates for LO phonons with a given momentum $\boldsymbol{q}_{\perp}\left(w_{-}\right.$differs from $w_{+}$by a factor of $\left.n_{\mathrm{LO}}\left(\boldsymbol{q}_{\perp}\right) /\left(n_{\mathrm{LO}}\left(\boldsymbol{q}_{\perp}\right)+1\right)\right)$.

In the same way we can write the balance equation for the electrons in the ground state

$$
\frac{\mathrm{d} n_{0}\left(\boldsymbol{k}_{1 \perp}\right)}{\mathrm{d} t}=\sum_{q_{\perp} \boldsymbol{k}_{0 \perp}}\left[w_{+} n_{1}\left(\boldsymbol{k}_{1 \perp}\right)-w_{-} n_{0}\left(\boldsymbol{k}_{0 \perp}\right)\right]-R_{0}\left(\boldsymbol{k}_{0 \perp}\right) n_{0}\left(\boldsymbol{k}_{0 \perp}\right) .
$$

To establish the kinetic equation for LO phonons we assume that the escape rate for LO phonons is negligible with respect to its decay rate:

$$
\frac{\mathrm{d} n_{\mathrm{LO}}\left(\boldsymbol{q}_{\perp}\right)}{\mathrm{d} t}=\sum_{\boldsymbol{q}_{\perp} \boldsymbol{k}_{0 \perp}}\left[w_{+} n_{1}\left(\boldsymbol{k}_{1 \perp}\right)-w_{-} n_{0}\left(\boldsymbol{k}_{0 \perp}\right)\right]-\alpha\left(\boldsymbol{q}_{\perp}\right) n_{\mathrm{LO}}\left(\boldsymbol{q}_{\perp}\right) .
$$

Here $\alpha\left(\boldsymbol{q}_{\perp}\right)=\gamma_{0}\left(1+n_{\mathrm{TA}}\right)$ is the decay rate for $\mathrm{LO}$ phonons. The factor $\left(1+n_{\mathrm{TA}}\right)$ takes into account the process of stimulated emission [12].

Finally, for the TA phonons we have

$$
\frac{\mathrm{d} n_{\mathrm{TA}}}{\mathrm{d} t}=\sum_{\boldsymbol{q}_{\perp}}\left[\alpha\left(\boldsymbol{q}_{\perp}\right) n_{\mathrm{LO}}\left(\boldsymbol{q}_{\perp}\right)\right]-n_{\mathrm{TA}} / \tau
$$

where $\tau^{-1}$ is the rate of escape from the well. For simplicity we do not distinguish between TA phonons with slightly different properties that appear due to the decay of LO phonons with different $\boldsymbol{q}_{\perp}$.

Due to the sums appearing in (14) to (17), they are integro-differential equations. To proceed further we have to make additional assumptions. We assume that $\delta \epsilon \ll \epsilon_{F}^{L}<\hbar \omega_{0}$ (which is satisfied near resonance, with the Fermi level of $15 \mathrm{meV}$ used in our calculations). The LO phonons are more efficiently generated if they have very small momenta in the perpendicular direction:

$$
\left|\boldsymbol{q}_{\perp}\right|=\left|\boldsymbol{k}_{1 \perp}-\boldsymbol{k}_{0 \perp}\right| \sim \frac{m^{*} \delta \epsilon}{\hbar^{2} k_{1 \perp}} .
$$

Furthermore, such phonons decay more slowly. It has been shown experimentally by de Paula et al [41] that confined LO phonons with non-zero momentum in GaAs heterostructures normally have lifetimes about half that of bulk LO phonons at the Brillouin zone centre.

Supposing that

$$
n_{\mathrm{LO}}\left(\boldsymbol{q}_{\perp}\right)=n_{\mathrm{LO}} \delta_{\boldsymbol{q}_{\perp}, \mathbf{0}}
$$

we can sum (14) over all possible directions of $\boldsymbol{k}_{0 \perp}$. It is convenient to define a vector $\boldsymbol{k}_{1 \perp}^{*}$ that has the modulus of $\boldsymbol{k}_{1 \perp}$ but is in the direction of $\boldsymbol{k}_{0 \perp}$ :

$$
\boldsymbol{k}_{1 \perp}^{*} \equiv \boldsymbol{k}_{0 \perp} \frac{k_{1 \perp}}{k_{0 \perp}} .
$$


Thus we get

$$
\begin{aligned}
\frac{\mathrm{d} n_{1}\left(\boldsymbol{k}_{1 \perp}\right)}{\mathrm{d} t}=G\left(\boldsymbol{k}_{1 \perp}\right)-n_{\mathrm{LO}} \sum_{\boldsymbol{k}_{0 \perp}} W\left(\boldsymbol{k}_{0 \perp}, \boldsymbol{k}_{1 \perp}^{*}\right)\left[n_{1}\left(\boldsymbol{k}_{1 \perp}^{*}\right)-n_{0}\left(\boldsymbol{k}_{0 \perp}\right)\right] \\
-\sum_{\boldsymbol{k}_{0 \perp}, \varphi} W\left(\boldsymbol{k}_{1 \perp}, \boldsymbol{k}_{0 \perp}\right) n_{1}\left(\boldsymbol{k}_{1 \perp}\right)-R_{1}\left(\boldsymbol{k}_{1 \perp}\right) n_{1}\left(\boldsymbol{k}_{1 \perp}\right)
\end{aligned}
$$

where $\varphi$ is the angle between $\boldsymbol{k}_{0 \perp}$ and $\boldsymbol{k}_{1 \perp}$, and $W\left(\boldsymbol{k}_{1 \perp}, \boldsymbol{k}_{0 \perp}\right)$ is given by formula (8) but without the factor $n_{\mathrm{LO}}+1$.

It is clear from (20) that $W\left(\boldsymbol{k}_{0 \perp}, \boldsymbol{k}_{1 \perp}^{*}\right)$ is independent of the direction of $\boldsymbol{k}_{1 \perp}$; thus (21) can be rewritten as

$$
\begin{aligned}
& \frac{\mathrm{d} n_{1}\left(k_{1 \perp}\right)}{\mathrm{d} t}=G\left(k_{1 \perp}\right)-R_{1}\left(k_{1 \perp}\right) n_{1}\left(k_{1 \perp}\right) \\
&-n_{\mathrm{LO}} w_{1}\left(k_{1 \perp}\right)\left[n_{1}\left(k_{1 \perp}\right)-n_{0}\left(\tilde{k}_{1 \perp}\right)\right]-w_{2}\left(k_{1 \perp}\right) n_{1}\left(k_{1 \perp}\right)
\end{aligned}
$$

where all of the quantities depend only upon the modulus $k_{1 \perp}$.

Here

$$
\begin{aligned}
w_{1}=\sum_{\boldsymbol{k}_{0 \perp}} W\left(\boldsymbol{k}_{0 \perp}, \boldsymbol{k}_{1 \perp}^{*}\right)=2 \pi \sum_{k_{0 \perp}} W\left(\boldsymbol{k}_{0 \perp}, \boldsymbol{k}_{1 \perp}^{*}\right) \\
=\frac{\pi e^{2}\left[I_{1} q_{z}+\left(2 m^{*} \delta \epsilon / \hbar^{2}\right) I_{2}\right]^{2}}{\epsilon^{*} m^{*} \omega_{0} d} \frac{\theta\left(\delta \epsilon+\hbar^{2} k_{1 \perp}^{2} /\left(2 m^{*}\right)\right)}{q_{z}^{2}+2 m^{*} \delta \epsilon / \hbar^{2}+2 k_{1 \perp}\left(k_{1 \perp}-\tilde{k}_{1 \perp}\right)}
\end{aligned}
$$

and

$$
\begin{aligned}
w_{2}=\sum_{k_{0 \perp}} W & \left(\boldsymbol{k}_{0 \perp}, \boldsymbol{k}_{1 \perp}\right) \\
= & \frac{\pi e^{2}\left[q_{z} I_{1}+\left(2 m^{*} \delta \epsilon / \hbar^{2}\right) I_{2}\right]^{2}}{\epsilon^{*} m^{*} \omega_{0} d} \frac{\theta\left(\delta \epsilon+\hbar^{2} k_{1 \perp}^{2} /\left(2 m^{*}\right)\right)}{\sqrt{\left(q_{z}^{2}+\left(k_{1 \perp}-\tilde{k}_{1 \perp}\right)^{2}\right)\left(q_{z}^{2}+\left(k_{1 \perp}+\tilde{k}_{1 \perp}\right)^{2}\right)}}
\end{aligned}
$$

(where $\tilde{k}_{1 \perp}$ was defined in (9)) are the out-of-equilibrium scattering rates. $w_{2}$ coincides with the first term in (11).

In the same way we get

$\frac{\mathrm{d} n_{0}\left(\tilde{k}_{1 \perp}\right)}{\mathrm{d} t}=-R_{0}\left(\tilde{k}_{1 \perp}\right) n_{0}\left(\tilde{k}_{1 \perp}\right)+w_{1}\left(\tilde{k}_{1 \perp}\right) n_{\mathrm{LO}}\left[n_{1}\left(k_{1 \perp}\right)-n_{0}\left(\tilde{k}_{1 \perp}\right)\right]+w_{2}\left(\tilde{k}_{1 \perp}\right) n_{1}\left(k_{1 \perp}\right)$.

By integrating over $\boldsymbol{q}_{\perp}$ in the balance equation (16), we get

$$
\frac{\mathrm{d} n_{\mathrm{LO}}}{\mathrm{d} t}=-\gamma_{0} n_{\mathrm{LO}}\left(1+n_{\mathrm{TA}}\right)+n_{\mathrm{LO}} \sum_{k_{1 \perp}} w_{1}\left(\tilde{k}_{1 \perp}\right)\left[n_{1}\left(k_{1 \perp}\right)-n_{0}\left(\tilde{k}_{1 \perp}\right)\right]+w_{2}\left(\tilde{k}_{1 \perp}\right) n_{1}\left(k_{1 \perp}\right) .
$$

The equation for TA phonons is

$$
\frac{\mathrm{d} n_{\mathrm{TA}}}{\mathrm{d} t}=-\frac{n_{\mathrm{TA}}}{\tau}+\gamma_{0} n_{\mathrm{LO}}\left(1+n_{\mathrm{TA}}\right) .
$$

Neglecting the dependence upon $k_{1 \perp}$ of $R_{0}$ and $R_{1}$ and the difference between $w_{1}$ and $w_{2}$, the kinetic equations can be simplified to the following forms:

$$
\begin{aligned}
& \frac{\mathrm{d} n_{1}}{\mathrm{~d} t}=G-w\left[n_{1}\left(n_{\mathrm{LO}}+1\right)-n_{0} n_{\mathrm{LO}}\right]-R_{1} n_{1} \\
& \frac{\mathrm{d} n_{0}}{\mathrm{~d} t}=w\left[n_{1}\left(n_{\mathrm{LO}}+1\right)-n_{0} n_{\mathrm{LO}}\right]-R_{0} n_{0}
\end{aligned}
$$




$$
\begin{aligned}
& \frac{\mathrm{d} n_{\mathrm{LO}}}{\mathrm{d} t}=w\left[n_{1}\left(n_{\mathrm{LO}}+1\right)-n_{0} n_{\mathrm{LO}}\right]-\gamma_{0}\left(1+n_{\mathrm{TA}}\right) n_{\mathrm{LO}} \\
& \frac{\mathrm{d} n_{\mathrm{TA}}}{\mathrm{d} t}=\gamma_{0}\left(1+n_{\mathrm{TA}}\right) n_{\mathrm{LO}}-n_{\mathrm{TA}} / \tau .
\end{aligned}
$$

In this work we limit ourselves to studying the stationary solutions of this system of equations. In the steady state, the equations can be rewritten as

$$
\begin{aligned}
& G=R_{0} n_{0}+R_{1} n_{1} \\
& R_{0} n_{0}=w\left[n_{1}\left(n_{\mathrm{LO}}+1\right)-n_{0} n_{\mathrm{LO}}\right] \\
& R_{0} n_{0}=\gamma_{0}\left(1+n_{\mathrm{TA}}\right) n_{\mathrm{LO}} \\
& n_{\mathrm{TA}} / \tau=\gamma_{0}\left(1+n_{\mathrm{TA}}\right) n_{\mathrm{LO}} .
\end{aligned}
$$

By taking $G, R_{0}, R_{1}, \gamma_{0}, w$, and $\tau$ as parameters independent of the populations, the kinetic equations can be easily solved. We can express all of the populations in terms of $n_{\mathrm{TA}}$ :

$$
\begin{aligned}
& n_{0}=\frac{n_{\mathrm{TA}}}{\tau R_{0}} \\
& n_{1}=\frac{G-n_{\mathrm{TA}} / \tau}{R_{1}} \\
& n_{\mathrm{LO}}=\frac{n_{\mathrm{TA}}}{\gamma_{0}\left(n_{\mathrm{TA}}+1\right) \tau}
\end{aligned}
$$

and then we get the following equation for $n_{\mathrm{TA}}$ :

$\left[1+\tau \gamma_{0}+\frac{\gamma_{0} \tau R_{1}}{w}+\frac{R_{1}}{R_{0}}\right] n_{\mathrm{TA}}^{2}+\left[\tau \gamma_{0}+\frac{\gamma_{0} \tau R_{1}}{w}-G \tau\left(\gamma_{0} \tau+1\right)\right] n_{\mathrm{TA}}-G \tau^{2} \gamma_{0}=0$

with only one positive root. In fact the energy levels in the well, and therefore the electric current, depend upon the accumulated charge inside the well. As a consequence, the current, the populations, and the potential profile have to be calculated self-consistently. Now we will describe the calculation of the parameters that appear in the kinetic equations.

\subsection{The electronic current}

The energy levels of the system are calculated by numerical diagonalization of the electronic profile. The site dependence of the local energies $e_{i}$ describes the potential profile including the effects of the applied bias and the charge accumulation in the well.

We have obtained the current circulating through the sample by using the simplest effective-mass approximation [42]:

$G=\pi S \int \frac{\mathrm{d}^{3} k}{(2 \pi)^{3}} \delta\left(\epsilon_{1}-\frac{\hbar^{2} k_{z}^{2}}{2 m^{*}}\right) \Gamma\left(k_{z}\right) v_{z}\left(k_{z}\right)\left[\theta\left(\epsilon_{F}^{L}-\frac{\hbar^{2} k_{z}^{2}}{2 m^{*}}\right)-n_{1} / N_{r}\right]$

where $S$ is the surface area of the device interfaces (we take $S=500 \mu \mathrm{m}^{2}$ ), $\Gamma\left(k_{z}\right)=$ $\left(2 \pi \hbar^{2} /\left(m^{*} d^{2}\right)\right) T_{L}, T_{L}$ is the transmittance of the left-hand barrier, $v_{z}$ is the electron velocity parallel to the current, and $N_{r}$ is the number of resonant states in the well, $N_{r} \sim S / a^{2}, a$ being the lattice constant. Our calculations (see figure 4, later) show that $n_{1} / N_{r} \ll 1$. It is straightforward then to see that this equation can be reduced to

$$
G=S \frac{m^{*}}{4 \pi \hbar^{3}} \theta\left(\epsilon_{F}^{L}-\epsilon_{1}\right)\left(\epsilon_{F}^{L}-\epsilon_{1}\right) \Gamma\left(\epsilon_{1}\right) \theta\left(\epsilon_{1}\right) .
$$

In this calculation we have neglected the width of the localized level at the well. Therefore the current vanishes if the first excited level is above the Fermi level or below the bottom of 
the conduction band of the emitter. For the barrier sizes described in section 1, we calculate the escape rates $R_{0}$ and $R_{1}$ from the transmittances. They vary slightly with the applied bias and are of the order of $R_{1}=0.05 \mathrm{ps}^{-1}$ and $R_{0}=0.007 \mathrm{ps}^{-1}$. From reference [12] we get $\gamma_{0}=0.11 \mathrm{ps}^{-1}$. We have also estimated $\tau \simeq 10 \mathrm{ps}$.

\subsection{The LO-phonon emission rate}

As we said at the beginning of this section, the strength of the LO-phonon emission rate was approximated as $w_{1} \sim w_{2}=w$. Also, these values depend on the electron momentum perpendicular to the current $\boldsymbol{k}_{1 \perp}$. We can see from formulae (23) and (24) that these values jump from zero to a finite value when $\delta \epsilon+\hbar^{2} k_{1 \perp}^{2} /\left(2 m^{*}\right)$ becomes positive. By averaging this function over $k_{1 \perp}$, we obtain a behaviour of $w$ as a function of $\delta \epsilon$ that rises sharply (but not vertically) from zero to an almost constant value $w_{0}$ over a region of width $\Delta$ $(\Delta \approx 0.7 \mathrm{meV})$. Therefore we approximate the emission rate $w$ as follows:

$$
w= \begin{cases}w_{0} \mathrm{e}^{-f(V)^{2}} & \text { for } f(V)<0 \\ w_{0} & \text { for } f(V) \geqslant 0\end{cases}
$$

where $f(V) \equiv\left(\delta \epsilon+\frac{1}{2}\left(\epsilon_{F}^{L}-\epsilon_{1}\right)\right) / \Delta$ is the average of the arguments of the step function in (23) and (24) divided by the width $\Delta$. It is a function of the applied bias $V$. The step height $w_{0}$ can be estimated as

$$
w_{0}=w_{1}\left(k_{1 \perp}=0\right)=w_{2}\left(k_{1 \perp}=0\right)=3^{1 / 2} \alpha_{F} \omega_{0}
$$

where

$$
\alpha_{F}=\frac{e^{2}}{\epsilon^{*}}\left(\frac{m^{*}}{2 \hbar^{3} \omega_{0}}\right)^{1 / 2}
$$

is the Fröhlich constant. With the values given by Pavesi and Guzzi [43] we get $\alpha_{F} \approx 0.068$ for GaAs. Therefore the estimated value is $w_{0} \approx 6.5 \mathrm{ps}^{-1}$. This value is of the same order as those reported by Skolnick et al [44] and Ferreira and Bastard [45].

Since $w_{0}$ appears to be much larger than all of the other relevant rates, its exact value is not very important. In fact, we can distinguish three different situations for $w$ varying with the applied bias $V$.

(i) In the region where $w=0$ ( or $G=0$ ), no phonon emission takes place. Therefore this situation is not interesting for the problem studied here.

(ii) The region where $w \sim w_{0}$.

We can see that in equation (39) - that is the only point of our calculation at which $w$ is used- $w$ appears only in the combination $\gamma_{0} \tau R_{1} / w$.

Using the parameters listed at the end of section 3.2, and a typical value of the electron input rate $G \sim 1000 \mathrm{ps}^{-1}$, we obtained $\gamma_{0} \tau R_{1} / w_{0} \sim 0.008$, which is negligible in comparison with $R_{1} / R_{0} \sim 7.1$ (in the first term of this expression) and even more irrelevant with respect to $G \tau\left(1+\gamma_{0} \tau\right) \sim 21000$, and $G \tau^{2} \gamma_{0} \sim 11000$ which appear in the two other terms of equation (39).

(iii) The small region in which $w$ is of the order of $\gamma_{0} \tau R_{1}$, which presents an instability that can be seen in figures 3-5, later. This instability will be discussed at the end of this article. 


\subsection{The effect of the charge accumulated in the well}

It is important also to consider the effect of the charge accumulated in the well on the LO-phonon emission. This effect could lead to instabilities that could turn out to make the operation of the device unreliable. Fortunately, it can be shown that the region of instabilities (although depending on the characteristics of the device) is very small.

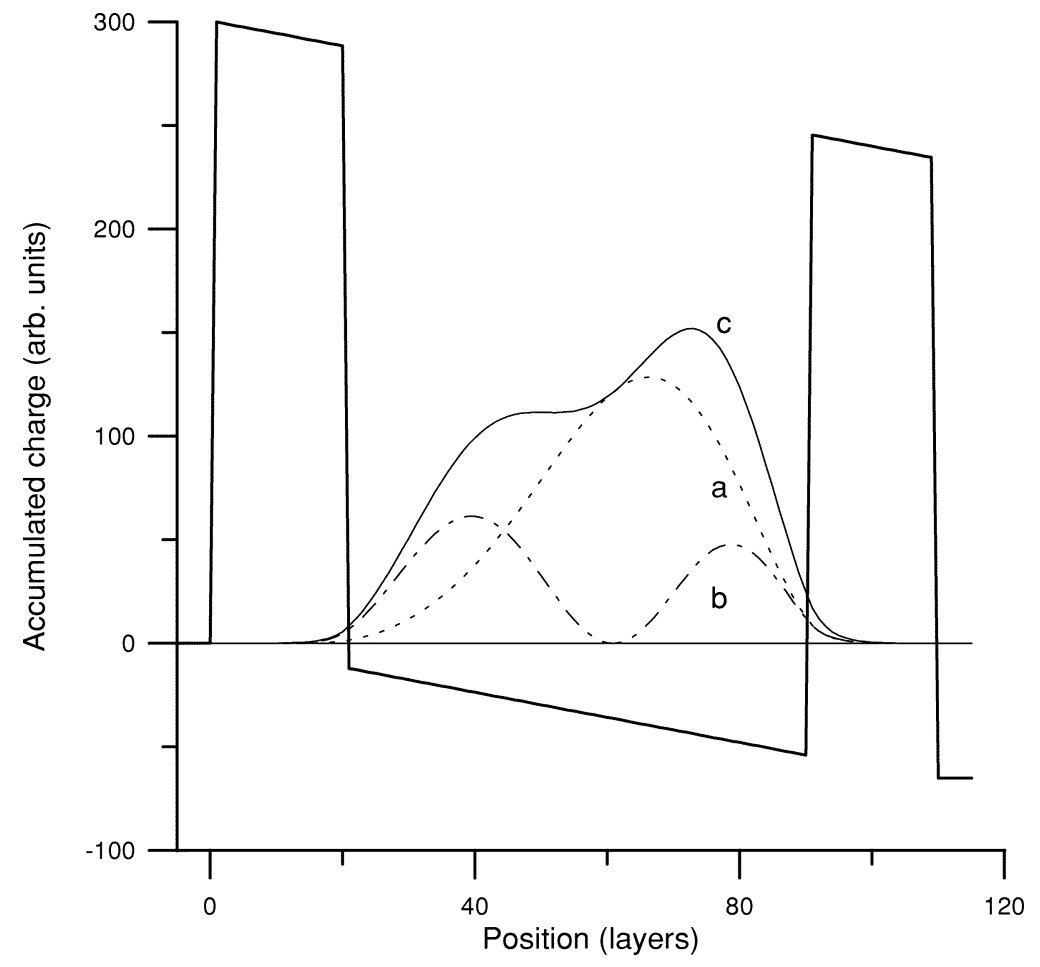

Figure 2. The spatial charge distribution. (a) For the ground state. (b) For the excited state. (c) The total charge.

The simplest way to consider the charge accumulation is to solve self-consistently equations which define the charge and potential profiles in the DBHS by assuming a uniform charge distribution inside the well. This can be justified a posteriori by adding the charge profiles of each level obtained by direct diagonalization, as is shown in figure 2. The equations relating the potential profile and the charge density across the DBHS are the Poisson equation

$$
\nabla^{2} \phi=\frac{4 \pi e}{\epsilon}\left(n-N_{d}\right)
$$

(where $\phi(z)$ is the electric potential, $\epsilon$ is the dielectric constant, $n$ is the density of electrons, and $N_{d}$ is the donor density) and the drift-diffusion equation describing the carrier distributions in the emitter and the collector:

$$
J_{E}+J_{D}=J_{T}
$$

where

$$
J_{E}=n(z) \eta \frac{\mathrm{d} \phi(z)}{\mathrm{d} z}
$$




$$
\begin{aligned}
& J_{D}=-D \frac{\mathrm{d} n(z)}{\mathrm{d} z} \\
& J_{T}=\frac{I}{S} .
\end{aligned}
$$

Here $\eta$ is the mobility, $D$ is the diffusion coefficient, and $I=-e G$ is the electric current.

The boundary conditions are

$$
\begin{aligned}
& \phi\left(-L_{1}\right)=0 \\
& \phi\left(L_{2}\right)=V .
\end{aligned}
$$

Here $L_{1}$ and $L_{2}$ are the emitter and collector lengths respectively, and $V$ is the applied potential.



Figure 3. The $I(V)$ characteristic calculated for a 20-70-20 DBHS. It shows an instability at the very beginning of the phonon emission. The current is expressed in electrons per ps. $\epsilon_{F}^{L}=15 \mathrm{meV}$.

The solution of this system is straightforward and yields an accumulation layer at the emitter and a depletion layer at the collector. The uniform charge distribution inside the well gives a parabolic contribution there. The rate equations have been calculated self-consistently using this potential profile, which is shown in figure 2. The parabolic contribution inside the well is too small to be noticed in the figure but is important to the study of the stability of the system. 


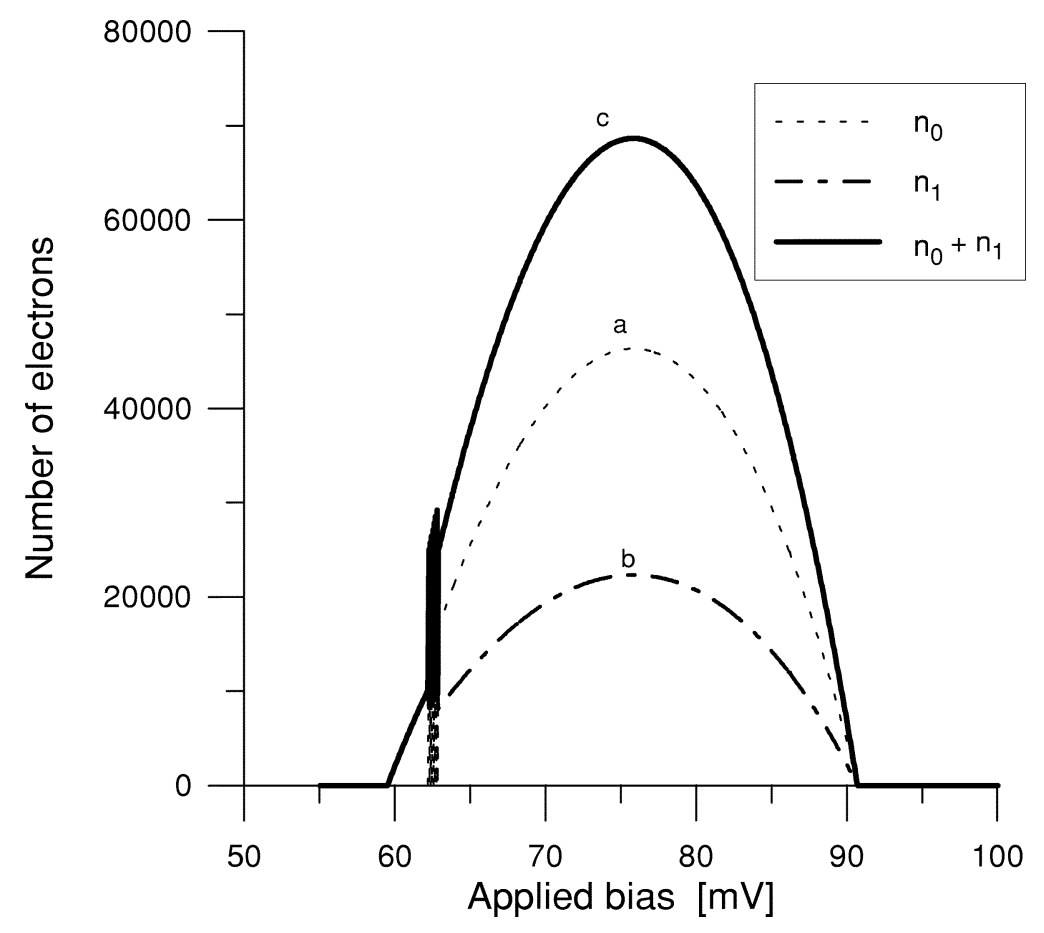

Figure 4. The electron populations in the well corresponding to the $I(V)$ characteristic of figure 3. (a) For the ground state. (b) For the excited state. (c) The total charge.

\subsection{The calculated results}

A typical calculated current-voltage characteristic is shown in figure 3 . The corresponding dependence of the electron populations in the well on the bias is presented in figure 4 . The electron-phonon interaction switches on at $V \approx 62 \mathrm{mV}$. The amount of charge in the well immediately increases, and this may lead to an instability of the current-voltage characteristics. Fortunately, for the symmetric DBHS this instability is negligible.

We have calculated the intensity of the acoustic phonon beam; it is typically of the order of 400 phonons per ps (see figure 5). In other words, $n_{\mathrm{TA}}$ is large enough to make our rough estimate of the phonon escape rate $\tau$ not especially crucial.

\section{Discussion and conclusions}

We have shown that, if nearly $\Gamma$-point $L O$ phonons generated by electron transitions in the well decay into a couple of (TA and $\widetilde{\mathrm{LO}}$ ) nearly L-point secondary phonons, and the secondary TA phonons escape from the well at a rate proportional to their group velocity, then it is possible to make the number of the TA phonons large compared to unity. This is basic for emission of coherent phonons. We have studied a particular case of a GaAsAlGaAs DBHS, but it is clear that the phenomenon of coherent sound generation could be produced in other structures. For example, in a well made of InP the size of the system has to be adjusted to the LO-phonon frequency of this material, and the phonons decay coherently by emitting a pair of LA phonons at the middle of the Brillouin zone [12].

The calculated emission intensity is of the same order as the intensity, in photons per 


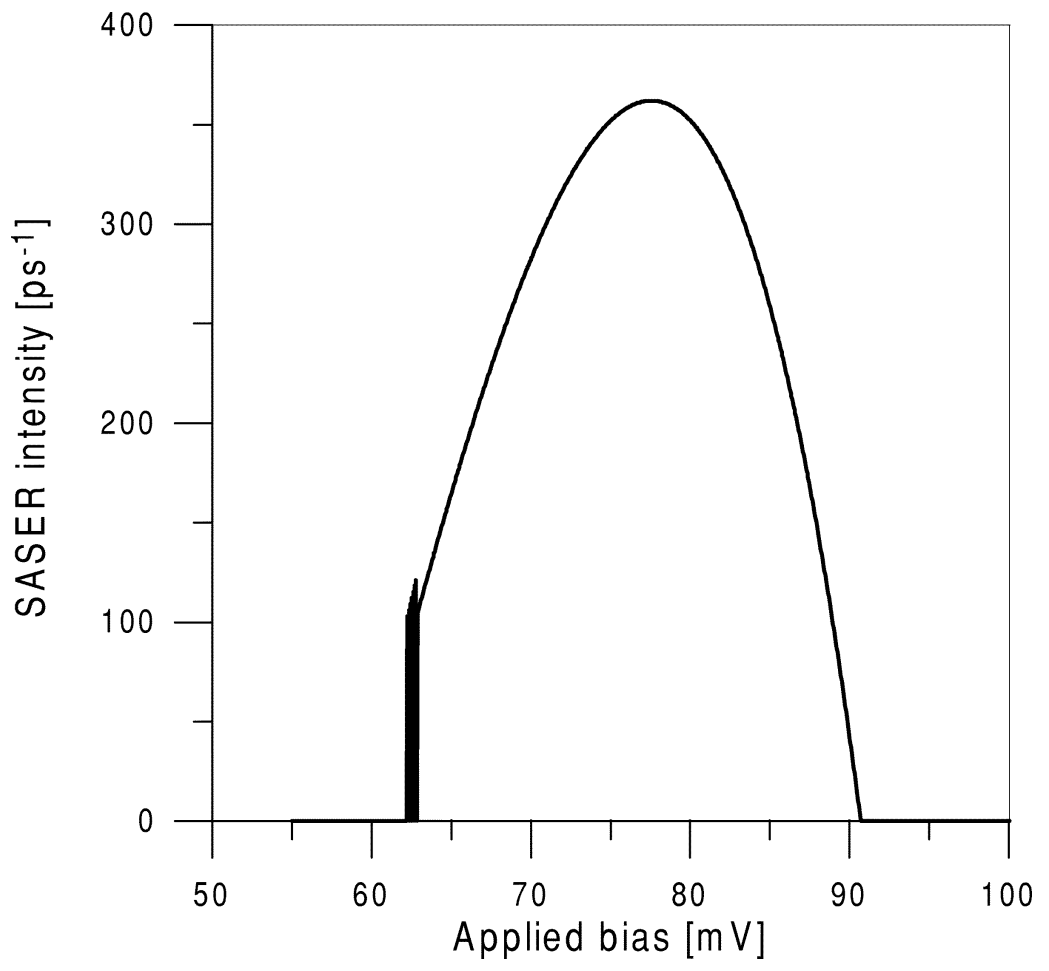

Figure 5. The phonon beam intensity measured in phonons per ps. The linear region could be useful for modulating the beam amplitude.

ps, of a standard He-Ne laser with a power of about $0.125 \mathrm{~mW}$ and a wavelength of about $6300 \AA$. Admittedly, as the energy of each photon is here 200 times greater than the phonon energy, the power of our device is very much lower.

Ultrasound is currently used for imaging, mainly for medical applications. Ultrasound equipment works at about $4 \mathrm{MHz}$, because the human body is composed basically of water. Some commercial instruments exploit the fact that the ultrasound is produced by a single source (a piezoelectric transducer), and therefore is coherent, to produce holograms of, for example, a baby inside its mother's uterus.

More recently, higher-frequency equipment has been used for research and industrial applications of acoustic imaging. Scanning acoustic microscopy (SAM) was extensively reviewed in a recent article [46], where one can find detailed descriptions of transmission and reflection acoustic microscopes, and images with a resolution higher than $0.4 \mu \mathrm{m}$, taken with $2 \mathrm{GHz}$ phonons.

In addition to the scanning acoustic microscopy, several other techniques are described in another recent review [47]: photoacoustic microscopy (PAM), scanning electron acoustic microscopy (SEAM), and scanning laser acoustic microscopy (SLAM). The theory of acoustic imaging is also reviewed, schematic diagrams of the different microscopes presented, and several industrial applications discussed in reference [47].

The pieces of equipment mentioned above work at up to $10 \mathrm{GHz}$, three orders of magnitude lower than the frequency of the phonon beam considered in the present paper. Therefore they have a limited theoretical resolution given by their wavelength, 1000 times greater than could be generated by a DBHS device. 
It is well known that coherence is not a requirement for making images. However, some techniques, such as holography, can only be put into practice using coherent beams.

To make acoustic nanoscopy possible, it would be necessary to have a coherent beam of ultra-short-wavelength phonons. To obtain images of a small structure one would need waves much shorter than the size of the details of the system. The acoustic phonon beam proposed here will have a wavelength smaller than $25 \AA$ (which is less by about a factor of 20 than for the device described in [22]), capable of 'seeing' in detail structures of about $50 \mathrm{~nm}$. If we try to see structures at the submicron scale by using light, we realize that the soft $\mathrm{x}$-rays with the same wavelength will have energies greater than $0.5 \mathrm{keV}$, which could affect the system to be studied. Besides, a coherent beam of soft x-rays is not available yet. Large synchrotron light sources will produce these beams soon, but they will be very expensive.

A potential application of the DBHS device is one in which the phonon beam is used to get a hologram of a nanostructure. To achieve this, it is necessary to build near it thin barriers of AlGaAs designed as semimirrors, placed at $45^{\circ}$ to split the beam and to force one half to pass through the part of the sample that we are interested in and the other to pass across a very pure GaAs reference. After that, we could use mirrors (made of thick AlGaAs barriers) and again semimirrors to superimpose them and record the interference pattern with a detector. This pattern could be processed in a computer to get a hologram of the sample showing the three-dimensional shape of the nanostructure.

Furthermore, we have shown that the amplitude of the phonon beam can be easily modulated by varying the incoming current. This permits us, for example, to use the phonon beam to transmit information from one component of a circuit to another over very short distances. These 'phonoelectronic' devices could work at lower distances and energies that the optoelectronic ones, thus improving the computing performance.

Another potential application is that of using the phonon beam and a 'phonoresist' (a compound with a soft mode close to the phonon beam frequency) for lithography at a mesoscopic scale. Once more, due to the short wavelength and low energy of this beam, this procedure could turn out to be better than the traditional methods. In summary, this device would offer a wide range of potential applications.

If the material was not sufficiently pure, scattering of TA phonons by impurities could limit the application of the device. Another shortcoming could arise from the noise due to the thermal phonon background if the temperature was not low enough.

As we have noted, when the electron-phonon interaction switches on (figure 3), the built-in charge in the well increases, because the effective rate of electron escape from the well decreases (note that $R_{0} \ll R_{1}$ ). This has two principal consequences. First, the intrinsic bistability, which occurs when the tunnelling takes place from a quantized level in the accumulation layer in the emitter [48, 49], is enhanced and the hysteresis loop in the current-voltage characteristics should become more pronounced. Secondly, one may expect instability of the $I(V)$ characteristics when the interaction switches on. The sharply increased charge in the well (figure 4) pushes the resonant level in the well out of resonance. A similar effect leading to a chaotic behaviour has been studied in references [50-52]. Our results concerning this instability and the possibility of chaotic behaviour of the device will be published elsewhere.

Lastly, let us briefly recall the approximations used and outline possible improvements of the above theory. First, the electron-phonon scattering rate was calculated in the Born approximation which does not take into account polaronic corrections. It would be possible to consider the tunnelling model in terms of the polaronic picture [53-55] including the decay of the modes composed. We are working along these lines. The formalism is too extensive 
to describe here. Our preliminary results [56] show that the qualitative behaviour of the device is not changed, even if some small quantitative differences appear in the characteristic curve and the SASER intensity. Secondly, the electron-phonon matrix elements have been taken as independent of the bias although the wavefunctions change together with the shape of the well. This may result in some corrections being required to, e.g., the value of the threshold bias. Finally, a more detailed model of the propagation of the secondary phonons across the interface would be desirable in view of the applications in acoustic nanoscopy.

\section{Acknowledgments}

This work was supported financially by the Brazilian agencies CNPq, CAPES, FAPERJ, and FINEP, by the Russian agency RFFI, the agency JNICT (Portugal), and by the multinational project Antorchas/Vitae/Andes, grant B-11487/4B005. HMP and MIV wish to acknowledge the hospitality of the ICTP, Trieste, Italy, where this work was completed.

\section{References}

[1] Capasso F and Datta S 1990 Phys. Today 4374

[2] Goldman V J, Tsui D C and Cunningham J E 1987 Phys. Rev. Lett. 581256

[3] Goldman V J, Tsui D C and Cunningham J E 1987 Phys. Rev. B 367635

[4] Leadbeater M L, Alves E S, Eaves L, Henini M, Hughes O H, Celeste A, Portal J C, Hill G and Pate M A 1989 Phys. Rev. B 393438

[5] Anda E V and Flores F 1991 J. Phys.: Condens. Matter 39087

[6] Wingreen N S, Jacobsen V W and Wilkins J W 1989 Phys. Rev. B 4011834

[7] Støvneng J A, Hauge E H, Lipavský P and Špička V 1991 Phys. Rev. B 4413595

[8] Lake R, Klimeck G and Datta S 1993 Phys. Rev. B 476427

[9] Lake R, Klimeck G, Anantram M P and Datta S 1993 Phys. Rev. B 4815132

[10] Jusserand B, Mallot F, Moison J-M and Le Roux G 1990 Appl. Phys. Lett. 57560

[11] Jacob J M, Kim D M, Bouchalkha A, Sony J J, Klem J F, Hou H, Tu C W and Morkoç H 1994 Solid State Commun. 91721

[12] Vallée F 1994 Phys. Rev. B 492460

[13] Ulbrich R G 1984 Nonequilibrium Phonon Dynamics (NATO ASI Series B, vol 124) (New York: Plenum) p 114

[14] Ulbrich R G, Narayanamurty V and Chin M A 1980 Phys. Rev. Lett. 451432

[15] Tang C L 1964 Phys. Rev. 134 A1166

[16] Tucker E B 1961 Phys. Rev. Lett. 6547

[17] Giordmaine J A and Kaiser W 1966 Phys. Rev. 144176

[18] Garmire E, Pandarese F and Townes C H 1963 Phys. Rev. Lett. 11160

[19] Haken H 1976 Quantum Field Theory of Solids, an Introduction (Amsterdam: North-Holland) p 218

[20] Prieur J-Y, Höhler R, Joffrin J and Devaud M 1993 Europhys. Lett. 24409

[21] Prieur J-Y, Devaud M, Joffrin J, Barre C, Stenger M and Chevallier M 1996 Physica B 219+220 235

[22] Basseras P, Gracewski S M, Wicks G W and Miller R J D 1990 J. Appl. Phys. 752761

[23] Damen E P N, Arts A F M and de Wijn H W 1995 Phys. Rev. Lett. 744249

[24] Zavtrak S T 1995 Phys. Rev. E 512480

[25] Zavtrak S T 1995 Phys. Rev. E 513767

[26] Zavtrak S T 1995 Zh. Tekh. Fiz. 65123 (Engl. Transl. 1995 Sov. Phys.-Tech. Phys. 40 589)

[27] Zavtrak S T 1996 J. Acoust. Soc. Am. 99730

[28] Doinikov A A and Zavtrak S T 1996 J. Acoust. Soc. Am. 993849

[29] Zavtrak S T and Volkov I V 1997 Zh. Tekh. Fiz. 6792 (Engl. Transl. 1997 Sov. Phys.-Tech. Phys. 42 406)

[30] Fokker P A, Dijkhuis J I and de Wijn H W 1997 Phys. Rev. B 552925

[31] Fokker P A, Melzer R S, Wang Y P, Dijkhuis J I and de Wijn H W 1997 Phys. Rev. B 552934

[32] Merlin R 1997 Solid State Commun. 102207

[33] Trallero-Giner C and Comas F 1988 Phys. Rev. B 374583

[34] Wendler L and Pechstedt R 1987 Phys. Status Solidi b 141129

[35] Wendler L and Haupt R 1987 Phys. Status Solidi b 143487 
[36] Wendler L, Haupt R, Bechstedt F, Rücker H and Enderline R 1988 Superlatt. Microstruct. 4577

[37] Huang K and Zhu B 1989 Phys. Rev. B 3813777

[38] Rücker H, Molinari E and Lugli P 1991 Phys. Rev. B 443463

[39] Lugli P, Molinari E and Rücker H 1991 Superlatt. Microstruct. 10471

[40] Rücker H, Molinari E and Lugli P 1992 Phys. Rev. B 456747

[41] de Paula A M, Maciel A C, Weber G, Ryan J F, Dawson P and Foxon C T 1992 Semicond. Sci. Technol. 7 8120

[42] Jonson M 1989 Phys. Rev. B 395924

[43] Pavesi L and Guzzi M 1994 J. Appl. Phys. 754779

[44] Skolnick M S, Hayes D G, Simmonds P E, Higgs A W, Smith G W, Hutchinson H J, Whitehouse C R, Eaves L, Henini M, Hughes O H, Leadbeater M L and Halliday D 1990 Phys. Rev. B 4110754

[45] Ferreira R and Bastard G 1989 Phys. Rev. B 401074

[46] Yu Z and Boseck S 1995 Rev. Mod. Phys. 67863

[47] Gilmore R S 1996 J. Phys. D: Appl. Phys. 291389

[48] Leadbeater M L, Alves E S, Eaves L, Henini M, Hughes O H, Sheard F W and Toombs G A 1988 Semicond. Sci. Technol. 31060

[49] Sheard F W and Toombs G A 1992 Appl. Phys. Lett. 521228

[50] Prescilla C, Jona-Lasinio G and Capasso F 1991 Phys. Rev. B 435200

[51] Jona-Lasinio G, Prescilla C and Capasso F 1992 Phys. Rev. Lett. 682269

[52] Orellana P, Anda E and Claro F 1997 Phys. Rev. Lett. 791118

[53] Anda E V, Makler S S, Pastawski H M and Barrera R G 1992 XII SLAFES (Pichidangui, Chile) abstracts

[54] Anda E V, Makler S S, Pastawski H M and Barrera R G 1994 Braz. J. Phys. 24330

[55] Bonča J and Trugman S A 1995 Phys. Rev. Lett. 752566

[56] Tuyarot D E, Makler S S, Anda E V and Vasilevskiy M I 1997 Superlatt. Microstruct. 22427 